\title{
A method of image processing with QR code ablated on rough and highly reflective metal surface by laser
}

\author{
Li Jianhua ${ }^{1}$, Shen $\mathrm{Zhi}^{1}{ }^{1, \mathrm{a}}$, Yan Chaoning ${ }^{1}$, Dong Nan ${ }^{1}$ and Liang Haimin ${ }^{2}$ \\ ${ }^{1}$ School of Mechanical \& Electronical Engineering, Lanzhou university of Technology,Lanzhou, Gansu 730050, China \\ ${ }^{2}$ China Aluminum Limited by Share Ltd Liancheng branch company,Lanzhou,730332, China
}

\begin{abstract}
For the purpose of solving the tough problem of the recognition of QR code which is marked on rough and highly reflective metal surface by laser, this work proposes a method of image processing based on multi-feature fusion. This method was requested to establish the integrated feature combined by color feature, texture feature and classify pixel points by means of k-means clustering, then optimize the image of QR code by morphology, Finally, this method was applied to the QR code Laser marking on the AL97 casting aluminum ingots to recognize, then compare with the accepted method OTSU algorithm, The experimental results show that the method is effective obviously.
\end{abstract}

\section{Instructions}

Laser ablation is an important method of Direct Part Marking(DPM) technology, which has the advantages of non-contact, high speed, low cost and high resolution[1]. It, direct ablation of $\mathrm{QR}$ code on material surface by laser, has become an inevitable trend for material identification, and has been widely used in metal material, glass, ceramics, plastics and other materials[2-5].

Metal is a common material in the manufacturing process, the surfaces of metal have the characteristics of texture, high reflection and uneven illumination, as a result, laser ablation of the QR code is very difficult to obtain high-quality images[6-7], thus affect the effects of subsequent QR code recognition. For this problem, Wang Juan proposed an improved DPM bar code localization algorithm based on the improved Smallest Univalue Segment Assimilating Nucleus(SUSAN) corner detection and the near-neighbor propagation clustering under the semi-supervisory mechanism for the different brightness and deformation of the QR code image of the PCB and metal parts[8].Liu Ning-zhong proposed an algorithm which uses inverse filtering to reverse-restore the bar code image as the core, it solves the problem that inaccurate detection location of bar code and the low recognition rate in the complex background[9]. Aiming at the problems of high brightness, blurring and distortion in DPM code of metal parts, Liu Zhi and Mei Hongfang proposed to combine image acquisition with homomorphic filtering and machine learning to enhance the fast positioning of bar code area[10], and Zheng H R et al proposed the method of homomorphic filtering combined with gradient projection to realize the rapid positioning of metal surface QR code[11]. considering the problems of QR code positioning in metal parts because of the complex background and uneven illumination, An accurate positioning method for $2 \mathrm{~d}$ bar code of complex background metal parts based on edge and level set was proposed by Guo Gaifang et al[12],Wang Jia jing et al proposed a method to recovery QR code's spilled information based on stochastic resonance algorithm for the problems of high light low contrast and partial exposure on metal surface[13]. In the background of the local high-light phenomenon of laser marking on the metal surface, the bar code information of the high light area are reconstructed by the bar code reconstruction method based on five-step reconstruction model[14].

The main starting points of the above research works are the optical field correction, bar code location and so on under the complex background, and these works have solved the difficulties in $2 \mathrm{~d}$ bar code recognition of some metal surface successfully in certain specific occasions. However, in practical applications, there are a class of metals with rough and highly reflective metal surfaces, such as cast aluminum, cast zinc, cast steel, cast nickel, cast lead, which are difficult to apply the above algorithms, and their surfaces not only have the characteristics of common metal texture and high reflective, but also have the problem of image noise and uneven surface, as a result, these affect the recognition effect seriously. Considering the above situations, we select the aluminum ingot casting as the research object to propose $\mathrm{QR}$ code preprocessing method based on comprehensive feature clustering of color and texture in this paper. This method makes use of the combination of color feature and texture feature to compose comprehensive feature, and uses K-means clustering to

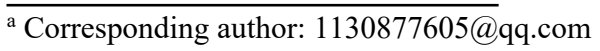


classify pixel, Finally, the QR code images with low contrast, uneven light intensity and image noise are optimized by morphological method. The experiments prove the effectiveness of the method.

\section{Problem descriptions about Image of QR code on casting aluminum}

Remelting casting aluminum ingots is the common metal raw material in industry, and QR code on its' surface is very bad in quality. As shown in Fig.1, there are not only texture and high-reflection in image of QR code on the surface of remelting casting aluminum ingots, but also noisy result from uneven surface. This phenomenon is common in casting metal material.

According to the test method of QR image in bar code standards, the quality of $\mathrm{QR}$ image can be described by contrast. Contrast formula is generally as follows:

$$
C=1-\frac{G_{R}}{G_{B}}
$$

In the formula, $\mathrm{C}$ is the contrast of $\mathrm{QR}$ image, $G_{R}$ is the average of minimal $10 \%$ pixels gray scale, $G_{B}$ is the average of maximal $10 \%$ pixels gray scale. QR image contrast (C) is used as QR symbol contrast (SC), A graded standard of $\mathrm{QR}$ image quality is established according to SC as follows:

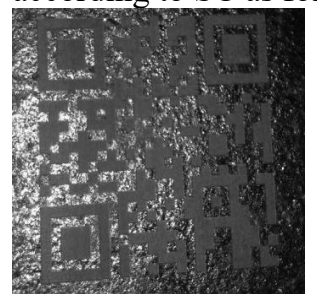

(a)

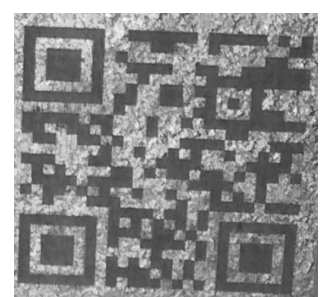

(b)

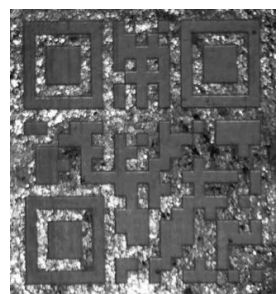

(c)

Fig. 1. QR code ablation by laser on Casting aluminum ingots. (a) $\mathrm{SC}=0.0416$; (b) $\mathrm{SC}=0.1258$; (c) $\mathrm{SC}=0.1379$

We randomly selected one hundred laser marking $\mathrm{QR}$ images on Casting aluminum ingots to get the value of $\mathrm{SC}$ and analyse, statistics about SC illustrate that most of them quality grades is $\mathrm{F}$ for $\mathrm{SC}$ range from 0.02 to 0.15 and the QR images quality is too low to scan as Fig.2.

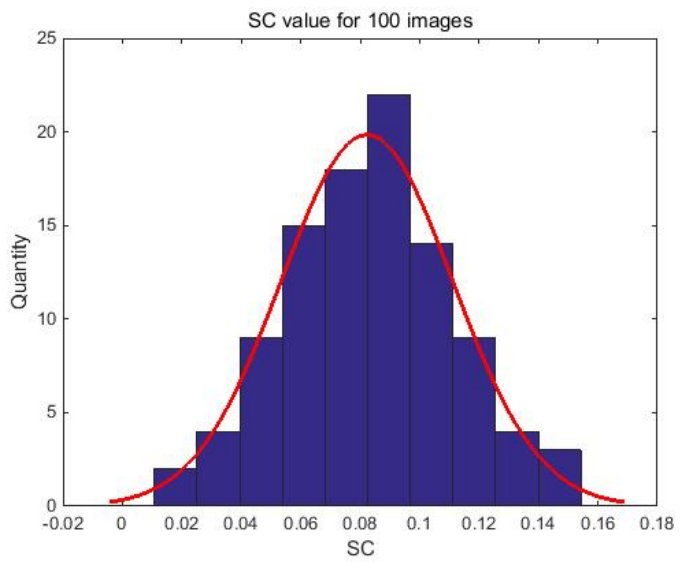

Fig. 2. QR code ablation by laser on Casting aluminum ingots

\section{Analysis of QR image process method}

$\mathrm{QR}$ code image processing could be considered as a segmentation between foreground and background. Supposing there is a QR code image with $\mathrm{N}$ pixels,after feature extraction and integration, we can get a feature vector with $\mathrm{d}$ dimensions for each pixel. Feature vectors of all pixels form feature vector set $\mathrm{X}, \mathrm{X}$ can be expressed as:

$$
\mathbf{X}=\left\{X_{i 1}, X_{i 2}, \cdots X_{i d}\right\}, i=1,2, \cdots \mathrm{N}
$$

The image needs to be divided into two categories: the foreground and the background, so the number of divided classes is $\mathrm{C}=2$. According to the overall probability density of the feature vector $\mathrm{Xi}$ of the Gauss mixed model, it can be expressed as:

\begin{tabular}{|c|c|c|c|}
\hline & $S C$ & Grade & Mark \\
\hline 0 & $S C \geqq .07$ & 4.0 & A \\
\hline 5 & $S C \geqq 0.5$ & 3.0 & B \\
\hline 0 & $S C \geqq 0.4$ & 2.0 & $\mathrm{C}$ \\
\hline 0 & $S C \geqq 0.2$ & 1.0 & $\mathrm{D}$ \\
\hline 0 & $S C<0.2$ & 0.0 & $\mathrm{~F}$ \\
\hline
\end{tabular}

Table1 Symbol contrast grade

$$
p\left(\mathbf{X}_{i}\right)=\sum_{j=1}^{c} P\left(\omega_{j}\right) p\left(\mathbf{X}_{i} \mid \omega_{j}\right)
$$

$\omega_{j}$ is the class $j$ of image segmentation. $P\left(\omega_{j}\right)$ represents the prior probability of class $j$ pixels in the whole image; $p\left(\mathbf{X}_{i} \mid \omega_{j}\right)$ is the 
class conditional probability density corresponding to the $\mathrm{Xi}$ of feature vectors under the condition of $\mathrm{J}$ class.

When the feature distributions of all the categories meet the Gaussian distribution, $\operatorname{Eq}(2)$ can be written as :

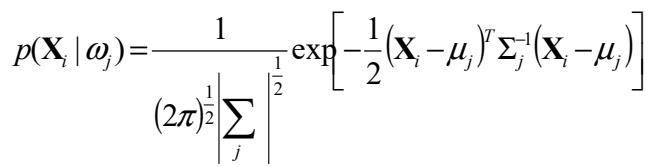

In the formula, $(\mu, \Sigma j)$ are the Gauss distribution parameters of class $j$, representing the mean vector and the covariance matrix, respectively.

According to the Bayes formula, the j-class's posterior probability of the pixel corresponding to the feature vector $\mathrm{Xi}$ is calculated as:

$$
p\left(\omega_{j} \mid \mathbf{X}_{i}\right)=\frac{P\left(\omega_{i}\right) p\left(\mathbf{X}_{i} \mid \omega_{j}\right)}{\sum_{j=1}^{C} P\left(\omega_{i}\right) p\left(\mathbf{X}_{i} \mid \omega_{i}\right)}
$$

Combining $\mathrm{Eq}(4)$ and $\mathrm{Eq}(5)$, we can get as follow:

$$
p\left(\omega_{j} \mid \mathbf{X}_{i}\right)=\frac{P\left(\omega_{i}\right) p\left(\mathbf{X}_{i} \mid \omega_{j}\right)}{p\left(\mathbf{X}_{i}\right)}
$$

On the basis of maximum likelihood function, the maximum expectation algorithm (EM) is used to get the $p\left(\omega_{m} \mid \mathbf{X}_{i}\right)$ of convergence by iteration.

If:

$$
p\left(\omega_{m} \mid \mathbf{X}_{i}\right)=\max _{j=1,2, \cdots, C} p\left(\omega_{j} \mid \mathbf{X}_{i}\right)
$$

Then:

$$
\mathbf{X}_{i} \in \omega_{m}
$$

Obviously, image segmentation can be achieved by approach of multi-feature integration from the above theoretical analysis.

\section{Image Processing Algorithm Based on Feature Fusion}

As the previous analysis, it can be feasible for $Q R$ code image recognition on rough and high brightness metal surface by the background segmentation method of multi -feature fusion. The $\mathrm{QR}$ code images have obvious differences in texture and color features. Therefore, the method of texture and color feature fusion is designed to divide the image. The specific steps are as follows:

(a) Normalize the size of the original image.

(b) Convert color space and extract color features.

(c)Enhance texture by DoG and extract texture features.

(d) Fuse color features and texture features.

(e) Use K-means algorithm for pixel clustering.

(f) Use morphological opening and closing method to optimize image and output binary images.

\subsection{Extraction of color features}

Analyzing Color feature of the $\mathrm{QR}$ code image on the surface of aluminum ingots and plotting RGB color space and Lab color space distribution, we can see scatter plot as Figure 3. Using principal component analysis, taking a set of orthogonal units of space units and normalizing each component set, finally the e distribution table can be obtained through the variance e of each component (Table 2). Obviously the distribution of Lab color space tends to be more clustered, and the level of interdependence of RGB components on each other is high, especially the brightness is determined by the three component ( $R$ G B) together and the similarities of different color space regions is different. So Lab color space is more suitable for color features.

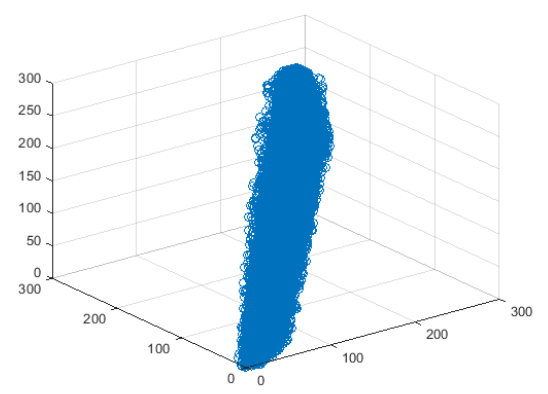

(a)

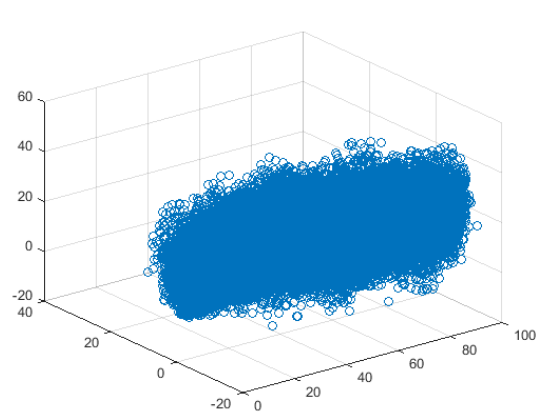

(b)

Fig. 3. Pixels in different Color model. (a) Pixels in RGB model; (b) Pixels in Lab model 
Table2 3 types e distribution

\begin{tabular}{clll}
\hline color space & $e_{1}$ & $e_{2}$ & $e_{3}$ \\
\hline $\mathbf{R G B}$ & 0.2495 & 0.2071 & 0.1383 \\
$\mathbf{L} * \mathbf{a} * \mathbf{b}$ & 0.2467 & 0.0342 & 0.0626 \\
\hline
\end{tabular}

Finally, a three-dimensional vector $\mathrm{C}(\mathrm{L}, \mathrm{a}, \mathrm{b})$ can be defined as a description of the color features of pixel $(\mathrm{x}, \mathrm{y})$, that is

$$
C(x, y)=(L, a, b)
$$

\subsection{Extraction of texture features}

The rough surface of aluminum ingot showed more high-frequency noise under natural lighting. But the laser ablation surfaces of aluminum ingot are flat and the reflectance was low, so they are mostly in the lowfrequency area. Otherwise, appropriate enhancement of image texture difference is conducive to get a good description of the texture features of $\mathrm{QR}$ code image on aluminum. The Difference of Gaussian(DoG) filter is similar to the Laplace of Gaussian(LoG) filter, and it is the optimal choice for detecting the intensity when ratio of the standard variance, $\sigma_{1}$ and $\sigma_{2}$, is 1.6: 1.0.

The mathematical model of DoG filter is

$$
\begin{aligned}
& \operatorname{DoG}(\mathrm{x}, y)=\frac{1}{2 \pi \sigma^{2}} e^{-\left(x^{2}+y^{2}\right) /\left(2 \sigma^{2}\right)}-\frac{1}{2 \pi K^{2} \sigma^{2}} e^{-\left(x^{2}+y^{2}\right) /\left(2 K^{2} \sigma^{2}\right)} \\
& =\mathrm{G}(x, y, \sigma)-\mathrm{G}(x, y, K \sigma)
\end{aligned}
$$

where $\mathrm{G}(\bullet)$ is Gaussian function, $\sigma_{1}$ and $\sigma_{2}$ are standard variance,. In order to simplify the calculation, the DoG filter can be regard as the difference of two discrete Gauss filters to generate Gauss convolution kernel with two standard variance $\sigma_{1}$ and $\sigma_{2}$. Convolution results are expressed as follows:

$$
\begin{array}{r}
g_{1}(x, y)=\mathrm{G}_{\sigma_{1}}(x, y) * f(x, y) \\
g_{2}(x, y)=\mathrm{G}_{\sigma_{2}}(x, y) * f(x, y)
\end{array}
$$

and

$$
\begin{aligned}
g_{1}(x, y)-g_{2}(x, y) & =\mathrm{G}_{\sigma_{1}}(x, y) * f(x, y)-\mathrm{G}_{\sigma_{2}}(x, y) * f(x, y) \\
& =\left(\mathrm{G}_{\sigma_{1}}(x, y)-\mathrm{G}_{\sigma_{2}}(x, y)\right) * f(x, y) \\
= & \mathrm{DoG} * f(x, y)
\end{aligned}
$$

For calculating faster and better, we attempted and found that the effects are pretty good for most images when $\sigma_{1}=1.6, \sigma_{2}=1.0$ and convolution kernel size is
$5 \times 5$, it reveals that the discrimination of foreground and background is greatly improved, and the problem of uneven distribution of light field can be overcome from Fig.4.

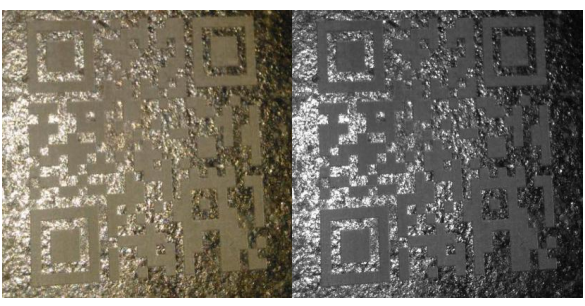

(a)

(b)

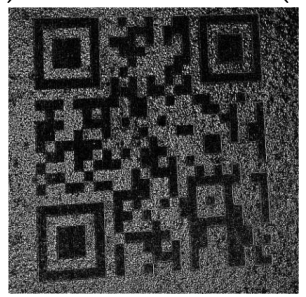

(c)

Fig. 4. texture comparison. (a) Origin image; (b) Gray image ;(c) Difference of Gaussian

The Co-Occurrence Matrix P is used to describe the QR code image texture. 14 kinds of texture feature can be extracted from $\mathrm{P}$, but only three descriptors which are entropy, inertia and energy can be distinguished with eyes. The specific definition is as follows:

entropy

$$
H=\sum_{i=0}^{L-1} \sum_{j=0}^{L-1} P(i, j) \lg P(i, j)
$$

Inertia

$$
I=\sum_{i=0}^{L-1} \sum_{j=0}^{L-1}(i-j)^{2} P(i, j)
$$

energy

$$
E=\sum_{i=0}^{L-1} \sum_{j=0}^{L-1}[P(i, j)]^{2}
$$

\subsection{Unsupervised classification of comprehensive features}

For the purpose of normalizing color feature vector and texture feature vector, all the pixel sub-features are extended to the to the 6-dimesion space,

$$
\mathbf{W}=\left\{\mathbf{X}\left(\mathbf{X}=\left(m_{1}, m_{2}, m_{3}, m_{4}, m_{3}, m_{6}\right) m \in\left[Q, M_{\text {mat }}\right] j=12, . .5,6\right\}\right.
$$

the values of the last 3 dimension of the color feature vector and first 3 dimension of he texture feature vector are all 0 after expansion, to ensure the different range of the feature mapping in the same range. Then, Calculate 
the maximum $\mathrm{M}_{\max }$ and average $\overline{\mathrm{m}}$ for each dimension, and multiply by $\left(\mathrm{M}_{\max }-\overline{\mathrm{m}}\right)^{2}$ in each dimension to highlight feature differences.

Considering that the contribution of color features and texture features to background segmentation is different, the method of constructing integrated Feature vector is as follow:

$$
\mathbf{S}(x, y)=\left(1-w_{t}\right) \overline{\mathbf{C}}(x, y)+w_{t} \overline{\mathbf{T}}(x, y)
$$

Where, $\mathbf{S}(x, y)$ is integrated feature, $\overline{\mathbf{C}}(x, y)$ and $\overline{\mathbf{T}}(x, y)$ are color feature and texture feature after normalization, $W_{t}$ is texture feature weight. 100 representative $\mathrm{QR}$ code images on aluminum ingot and corresponding binary images processed manually were used to establish training atlas, and LMS (least squares) method was used for offline learning and training. It can get $W_{t}=0.731$.

Clustering the Integrated features by K-means. The set $\boldsymbol{\Omega}$ containing n samples can be expressed as follow:

$$
\mathbf{\Omega}=\left\{\mathbf{X} \mid \mathbf{X}=\left(x_{i 1}, x_{i 2}, x_{i 3}, x_{i 4}, x_{i 5}, x_{i 6}\right), i=1,2, \ldots, n\right\}
$$

Where, $\mathbf{X}=\left(x_{i 1}, x_{i 2}, x_{i 3}, x_{i 4}, x_{i 5}, x_{i 6}\right)$ is a vector with 6 dimensions, and it represents the integrated feature vector of the pixel $i$.

The center of $\mathrm{n}$ samples can be expressed as follow:

$$
\mathbf{C}_{j}=\left[c_{j 1}, c_{j 2}, c_{j 3}, c_{j 4}, c_{j 5}, c_{j 6}\right], j=1,2
$$

Where $\mathbf{C}_{j}$ is the center of class $j$, and $\mathrm{K}$ is the number of pixel classes. The distance $\operatorname{dis}\left(\mathbf{X}_{i}, \mathbf{C}_{j}\right)$ between integrated feature vector $\mathbf{C}_{i}$ and $\mathbf{X}_{j}$ can be described as Euclid distance as follow:

$$
\operatorname{dis}\left(\mathbf{X}_{i}, \mathbf{C}_{j}\right)=\sqrt{\sum_{l=1}^{6}\left(\mathbf{X}_{i l}-\mathbf{C}_{j l}\right)^{2}}, i=1,2, \ldots, n ; j=1,2
$$

Define the center $\mathbf{C}_{j}$ of the $\mathrm{j}$-th class as follow:

$\mathbf{C}_{j}=\frac{1}{N\left(\phi_{j}\right)} \sum_{X_{i} \in \phi_{j}} X_{i}, j=1,2, \quad i=1,2, \cdots, n$

Where, $\phi_{j}$ is the set of class $j$, and $N\left(\phi_{j}\right)$ is the vector number of $\phi_{j}$.

Use the Sum of the Squared Error

(SSE) as objective function:

$$
S S E=\sum_{j=1}^{2} \sum_{x_{i} \in \phi_{j}} \operatorname{dis}\left(\mathbf{X}_{i}, \mathbf{C}_{j}\right)
$$

The integrated features in Eq.17 are used as the features of pixels, the value of center points $\mathrm{K}$ in $\mathrm{K}$ means is set to 2, and iterating clustering until SSE is stable. The pixels in first class are set to 0 and the pixels of second class are set to 1 . The binary images (Figure 5) are a clustering result.

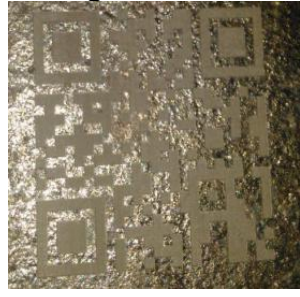

(a)

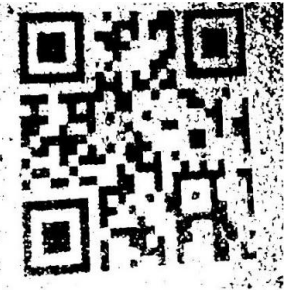

(b)
Fig. 5. Result of processing . (a) Origin image;(b)Result of Kmeans

In order to solve the problem of image discontinuity result from wrong clustering to some pixels, we need to improve the binary images Fig.4b by morphological methods. Through many experiments we found that it is best to use square kernel with size of $15 \times 15$ to do morphological opening and closing operation for the image Fig.4b with size of $653 \times 673$. The final result after morphological operation is shown in Fig.6.

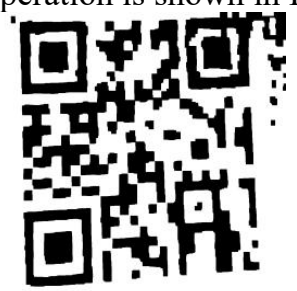

Fig. 6. final result

\section{Experiment and statistics}

The method proposed in the previous section is tested and verified. Pulsed fiber laser is used in experiment to direct ablation on the rough surface of aluminum ingots (AL99.70) to get 200 different QR code with size of $40 \mathrm{~mm}^{*} 40 \mathrm{~mm}$, then acquire $400 \mathrm{QR}$ Code images with size of 653X673 at different angles under natural light and flash light with OV9650 camera.

Then we use the reprocessing method proposed in this paper to process experimental samples, and select OTSU algorithm for comparison, table 3 shows the parts of experimental results, the recognition effect of the first part corresponds to OTSU recognition results, the second part corresponds to method proposed in this paper. $\times$ represents that it is unable to recognize, $\checkmark$ represents that it is Recognizable.

Table3 Parts of experimental result

\begin{tabular}{|l|l|l|l|}
\hline Samples & OTSU & $\begin{array}{l}\text { Feature } \\
\text { Fusion }\end{array}$ & effects \\
\hline
\end{tabular}




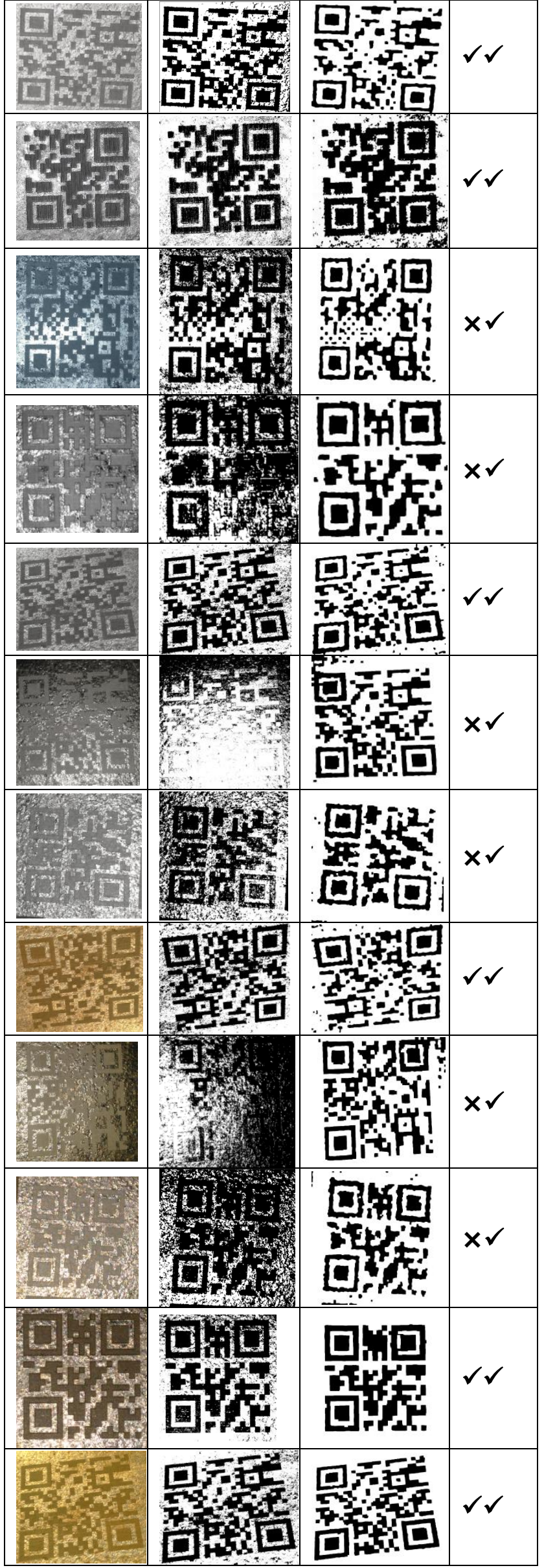

According to the experimental statistics, the recognition rate after OTSU treatment is about $50 \%$, and the recognition rate of this method is nearly $100 \%$ after processing. It is obvious that this method has an obvious advantage on the two-dimensional code image on dealing with rough and highly bright metal surface.

\section{6 conclusion}

Through theoretical analysis and experimental verification, we can draw the following conclusions:

(a) Based on the theory of mixed Gauss model, the feasibility of Foreground Background Segmentation under multi-feature fusion method is verified.

(b) Through calculation and experiment, the recognition rate of QR code is nearly $100 \%$ when the center number $\mathrm{k}$ is 2 , the texture feature weight $\omega_{t}$ is 0.731 , the size of the square core is $15 \times 15$, the actual size of the QR code is $40 \mathrm{~mm} \times 40 \mathrm{~mm}$ and the size of $\mathrm{QR}$ code image is $653 \times 673$.

(c) For the QR code recognition problems on rough and highly reflective metal surfaces, we propose an image processing algorithm based on multi-feature fusion and morphological optimization, and have a good recognition effect in the experiment, and it has a certain reference value to this class of metals whose surfaces are rough and highly reflective. Otherwise, this method has more advantages comparing with the OTSU algorithm

\section{Acknowledgment}

This work was supported by the Innovation Team Project of Chinese Ministry of Education (No. IRT1140).

\section{References}

1. J. Qi,K.L. Wang,Y.M. Zhu. A study on the laser marking process of stainless steel [J]. Journal of Materials Processing Tech.139 (1)(2003).

2. Noor Y M, Tam S C, Lim L E N, et al. A review of the Nd: YAG laser marking of plastic and ceramic IC packages [J]. Journal of Materials Processing Technology, 42 (1):95-133(1994).

3. Dumont $\mathrm{T}$, Lippert $\mathrm{T}$, Wokaun $\mathrm{A}$, et al. Laser writing of 2D data matrices in glass [J]. Thin Solid Films, s 453-454 (2):42-45(2004).

4. L Chitu, R Cernat, I Bucatica,et al. Improved Technologies for Marking of Different Materials[J].Laser Physics, 13 (8):1108-1111(2003).

5. Jangsombatsiri W, Porter J D. Laser Direct-Part Marking of Data Matrix Symbols on Carbon Steel Substrates [J]. Journal of Manufacturing Science \& Engineering, 129 (129):583-591(2007).

6. Wang Wei,HE Weiping,Lei Lei,et al.Speedy Location of 2D Bargion Under Complicated Metal Background $[\mathrm{J}]$. Journal of tianjin university, 46 (06):531-538(2013). 
7. Wang Wei,He Weiping,Lei lei,et al.2-D Bar Code Data Extraction on Metal Parts [J].Journal of Computer-Aided Design \& Computer Graphics, 24 (05):612-619(2012).

8. Wang Juan,Wang Ping.Direct part Mark Barcode Location Method [J].Journal of Computer-Aided Design \& Computer Graphics,26(07):11591166(2014).

9. Liu Ningzhong,Su Jun, Sun Han. QR Code Detection and Recognition Algorithm for Industrial Control [J]. Acta Electronica Sinica,39 (10):24592463(2011).

10. LIU Zhi,HONG Meifang. Research on the key technologies of rapid positioning on DPM barcode [J].Journal of Zhejiang University of Technology,44 (01):28-33(2016).

11. Zheng H R, Shan B, Liu Z. Locating Method of DPM 2D Barcode Image [J]. Advanced Materials Research, 989-994:3865-3867(2014) .

12. GUO Gaifang,HE Weiping,LI Xiashuang,et al.Accurate Localization of 2D Barcode Marked in Mental Parts Under Complex Background Based on Edge an $[\mathrm{J}]$. Journal of Shanghai Jiaotong University,51(01):90-97(2017).

13. WANG Jiajing,ZHANG Shusheng,HE Weiping.2D Bar Code Recovery in Highlight Area of Metal Parts[J].Journal of Shanghai Jiaotong University,47 (12):1836-1841(2013)

14. FANG Xinxin,LI Jianmei,LU Changhou, et al. Highlight area reconstruction of Data Matrix barcodes marked on metal surface [J].Computer Engineering and Applications,52 (16):205209(2016). 